\title{
On the flat transverse momentum dependence of the single-spin asymmetry in inclusive neutral pion production
}

\author{
S.M. Troshin, N.E. Tyurin \\ Institute for High Energy Physics, \\ Protvino, Moscow Region, 142281, Russia
}

\begin{abstract}
We discuss recent experimental results from RHIC where the flat transverse momentum dependence of a single-spin asymmetry has been found in the inclusive production of neutral pions. This dependence takes place in a wide region of the transverse momenta up to $p_{T}=10 \mathrm{GeV} / \mathrm{c}$. We emphasize that similar dependence has been predicted in the nonperturbative spin filtering mechanism for the single-spin asymmetries in hadron interactions and present some implications for this mechanism from the new experimental data.
\end{abstract}




\section{Introduction}

It should be noted that decrease with transverse momentum $p_{T}$ of single-spin asymmetries (SSA) is a common feature of perturbative QCD approaches including those based on account for the various modifications implemented into the calculation scheme originally grounded on collinear factorization. The most recent progress in this field is described in [1, 2]. The decreasing dependence has not been directly observed experimentally, but the experimental data were not very conclusive due to large statistcal errors. However, the existing data are consistent with the flat transverse dependence of SSA in inclusive processes. This conclusion is valid for the old data on of $\Lambda$-hyperon polarization [3], for example, and for the most recent data obtained at RHIC [4]. It is essential that the new data cover the wide region of the transverse momentum values up to $p_{T} \simeq 10 \mathrm{GeV} / \mathrm{c}$. In [6] it was noted that experimental data at higher values of $p_{T}$ would be needed to perform a more conclusive test of various pQCD theoretical approaches and their predictions. The model proposed in [6] provided flat $p_{T}$-dependence of SSA. The new data which have appeared very recently [4, 5] are consistent with the observed earlier trend, i.e. flat dependence on transverse momenta can be extended to the region of higher values of $p_{T}$. Since such high values of transverse momenta have been reached experimentally, one forced to conclude that the mechanism of the SSA's generation can have a nonperturbative origin. Of course, presence of the significant statistical errors in the current experimental data (cf. e.g [4, 5]) is the serious obstacle on the way of derivation of a completely unambiguous final conclusion on the impossibility of a decreasing dependence of the SSA with $p_{T}$.

\section{Highlights of the filtering mechanism of SSA genera- tion and new large- $p_{T}$ experimental data}

A nonperturbative QCD dynamics is closely interrelated with the two well-known phenomena, namely, color confinement and spontaneous breaking of chiral symmetry $(\chi \mathrm{SB})($ cf. e.g. [7]). The $\chi \mathrm{SB}-$ mechanism resulting in transition of current into constituent quarks is directly responsible for generation of their masses and appearance of quark condensates. Constituent quarks are colored objects, they appear to be quasiparticles and a hadron is often represented as a loosely bounded system of the constituent quarks. Simultaneously, the Goldstone bosons which are the excitations of the condensates appear and mediate interactions of the constituent quarks.

This interaction is mainly due to a pseudoscalar pion field and has therefore a spin-flip nature. Spin states filtering results from the unitarization process in the 
$s$-channel and connects SSA with asymmetries in the position (impact parameter) space [6].

The common features of SSA measurements at RHIC and Tevatron (linear increase of asymmetry with $x_{F}$ and flat transverse momentum dependence at large transverse momentum, $p_{T}>1 \mathrm{GeV} / \mathrm{c}$ ) are reproduced and described in the framework of the semiclassical picture based on the further development of the chiral quark model suggested in [8] and results of its use for the treatment of the polarized and unpolarized inclusive cross-sections including those obtained at RHIC [9].

We summarize now the essential features of the mechanism. Valence constituent quarks are scattered simultaneously (due to strong coupling with Goldstone bosons) and in a quasi-independent way by the effective strong field. In the initial state of the reaction $p p_{\uparrow} \rightarrow \pi^{0} X$ the proton is polarized and for the wave function of the proton we use the simple SU(6) model. The constituent quark $Q_{\uparrow}$ with transverse up spin fluctuates into Goldstone boson and another constituent quark $Q_{\downarrow}^{\prime}$ with down spin, performing spin-flip transition [10]:

$$
Q_{\uparrow} \rightarrow G B+Q_{\downarrow}^{\prime}
$$

It should be noted that $\pi^{0}$-fluctuation of constituent quark does not change its flavor and color. Assuming the equal probabilities of the processes with $U$ and $D$ quarks:

$$
U_{\uparrow, \downarrow} \rightarrow \pi^{0}+U_{\downarrow, \uparrow} \quad \text { and } \quad D_{\uparrow, \downarrow} \rightarrow \pi^{0}+D_{\downarrow, \uparrow},
$$

the production of $\pi^{0}$ by the polarized proton $p_{\uparrow}$ in this simple $S U(6)$ picture can be treated as a result of the fluctuation of the constituent quark $Q_{\uparrow}(Q=U$ or $D$ ) in the effective field into the system $\pi^{0}+Q_{\downarrow}$ (Fig. 1). Since the total angular

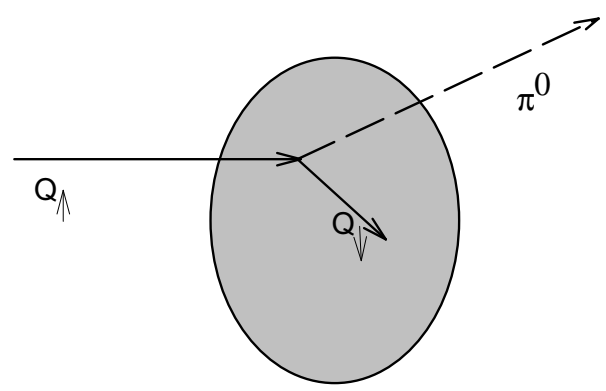

Figure 1: Chiral mechanism of $\pi^{0}$-production in polarized proton-proton interaction.

momentum is conserved, the compensation of quark spin flip should occur, i.e. to compensate quark spin flip $\delta \mathbf{S}$, an orbital angular momentum $\delta \mathbf{L}=-\delta \mathbf{S}$ should 
be associated with the final state of reaction (1). The introduction of $\delta \mathbf{L}$ implies a shift in the impact parameter value of the pion $\pi^{0}$ :

$$
\delta \mathbf{S} \Rightarrow \delta \mathbf{L} \Rightarrow \delta \tilde{\mathbf{b}}
$$

Note, that outside the hadron interior the Goldstone bosons are the usual pions and kaons. Due to different strengths of interaction at the different impact distances, i.e.

$$
\begin{aligned}
& p_{\uparrow} \Rightarrow Q_{\uparrow} \rightarrow \pi^{0}+Q_{\downarrow} \Rightarrow \quad-\delta \tilde{\mathbf{b}}, \\
& p_{\downarrow} \Rightarrow Q_{\downarrow} \rightarrow \pi^{0}+Q_{\uparrow} \Rightarrow+\delta \tilde{\mathbf{b}} .
\end{aligned}
$$

the processes of transition $Q_{\uparrow}$ and $Q_{\downarrow}$ to $\pi^{0}$ will have different probabilities. It eventually leads to nonzero asymmetry $A_{N}\left(\pi^{0}\right)$. When the shift in impact parameter is negative, $-\delta \tilde{\mathbf{b}}$, the interaction is stronger than that with the positive shift, $+\delta \tilde{\mathbf{b}}$, and therefore the asymmetry $A_{N}\left(\pi^{0}\right)$ is positive too. The shift in $\tilde{\mathbf{b}}$ (the impact parameter of final pion) is correlated with the shift of the impact parameter of the collision according to the relation between impact parameters in the multiparticle production process:

$$
\mathbf{b}=\sum_{i} x_{i} \tilde{\mathbf{b}}_{i}
$$

The production of $\pi^{0}$ with impact parameter $\tilde{\mathbf{b}}$ is considered in the fragmentation region, i.e. at large $x_{F}$ the approximate relation

$$
\mathbf{b} \simeq x_{F} \tilde{\mathbf{b}}
$$

which results from Eq. (4) has been used with additional assumption on the small values of Feynman $x_{F}$ for the other particles.

It should be noted that direction of spin of the proton is chosen to be along or opposite to the impact parameter vector $\mathbf{b}$. The integration over the asimuthal angle $\varphi$ selects therefore the finctions $I$ (see below) corresponding to the respective directions of the proton spin. The mechanism is illustrated in Fig. 2.

An essential feature of the mechanism is an account of unitarity in the direct channel of reaction. The corresponding formulas for inclusive cross-sections of the process

$$
p^{\uparrow, \downarrow}+p \rightarrow \pi^{0}+X
$$

have been obtained in [11]:

$$
d \sigma^{\uparrow, \downarrow} / d \xi=8 \pi \int_{0}^{\infty} b d b I^{\uparrow, \downarrow}(s, b, \xi) /|1-i U(s, b)|^{2},
$$




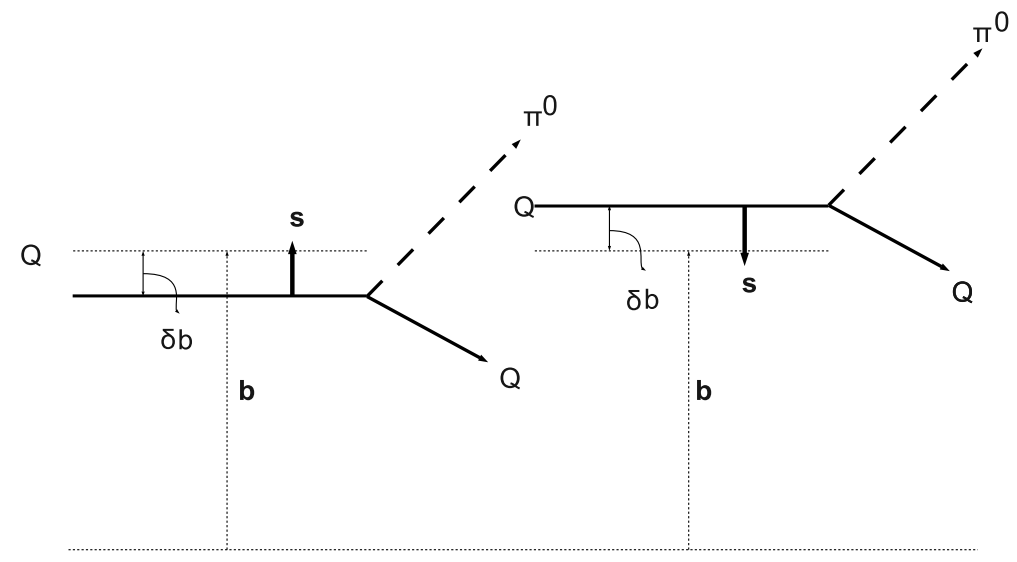

Figure 2: Schematic illustration of the SSA's generation mechanism due to spin flip of the constituent quark Q.

$b$ is the collison impact parameter. The function $U(s, b)$ is the generalized reaction matrix (averaged over initial spin states) which is determined by the basic dynamics of the elastic scattering. The elastic scattering amplitude in the impact parameter representation $F(s, b)$ is then given [8] by the relation:

$$
F(s, b)=U(s, b) /[1-i U(s, b)] .
$$

The equation (7) allows one to obey unitarity for the elastic scattering amplitude provided the inequality $\operatorname{Im} U(s, b) \geq 0$ takes place. The model [8] has been used for construction of the functional dependence of $U(s, b)$, namely, this function was chosen as a product of the factors corresponding to the averaged amplitudes of the individual valence quarks. The strong interaction radius of the quarks is determined by its mass,

$$
r_{Q}=\zeta / m_{Q}
$$

The parameter $\zeta$ was extracted from the experimental data for the differential cross-section of the elastic $p p$-scattering. In the region of medium values of $t$ this model provides[8] the familiar Orear-type behavior:

$$
\frac{d \sigma}{d t} \sim \exp \left(-\frac{2 \pi \zeta}{M} \sqrt{-t}\right)
$$


where $M$ is equal to the total mass of the constituent quarks in the two colliding protons, i.e. $M=6 m_{Q} \simeq 2 \mathrm{GeV} / \mathrm{c}$, and the value of parameter $\zeta \simeq 2$ since from the experimental data $m_{Q} / \zeta=150-200 \mathrm{MeV}$ and to reproduce the standard constituent quark masses the value of $\zeta$ should be around 2 .

The functions $I^{\uparrow, \downarrow}$ in Eq. (6) can be expressed through the functions $U_{n}^{\uparrow, \downarrow}-$ the multiparticle analogs of the function $U[11]$ in the polarized case. The set of the kinematical variables $\xi\left(x_{F}\right.$ and $p_{T}$ for example) describe the state of the produced pion.

We assume that the shift $\delta \tilde{b}$ can be connected with the radius of quark interaction $r_{Q}^{f l i p}$, which is responsible for the quark transition flipping its spin, i.e.:

$$
\delta \tilde{b} \simeq r_{Q}^{f l i p} .
$$

Asymmetry $A_{N}$ can be written in terms of the functions $I_{-}, I_{0}$ and $U$ :

$$
A_{N}(s, \xi)=\frac{\int_{0}^{\infty} b d b I_{-}(s, b, \xi) /|1-i U(s, b)|^{2}}{2 \int_{0}^{\infty} b d b I_{0}(s, b, \xi) /|1-i U(s, b)|^{2}},
$$

where $I_{0}=1 / 2\left(I^{\uparrow}+I^{\downarrow}\right)$ and $I_{-}=\left(I^{\uparrow}-I^{\downarrow}\right)$ and $I_{0}$ obey the sum rule

$$
\int I_{0}(s, b, \xi) d \xi=\bar{n}(s, b) \operatorname{Im} U(s, b),
$$

here $\bar{n}(s, b)$ stands for the mean multiplicity in the impact parameter representation.

With the above relation for the shift in the impact parameter due to the transition flipping quark spin the following expression for asymmetry $A_{N}^{\pi^{0}}$ can be written

$$
A_{N}^{\pi^{0}}(s, \xi) \simeq-x_{F} r_{Q}^{f l i p} \frac{1}{3} \frac{\int_{0}^{\infty} b d b I_{0}^{\prime}(s, b, \xi) d b /|1-i U(s, b)|^{2}}{\int_{0}^{\infty} b d b I_{0}(s, b, \xi) /|1-i U(s, b)|^{2}}
$$

where $I_{0}^{\prime}(s, b, \xi)=d I_{0}(s, b, \xi) / d b$. It is evident that $A_{N}^{\pi^{0}}(s, \xi)$ should be positive because $I_{0}^{\prime}(s, b, \xi)<0$.

In the model [ [8] the function $U(s, b)$ is chosen to be a product of the averaged quark amplitudes under assumption of the the quasi-independence of valence constituent quark scattering in the self-consistent mean field. The function $U(s, b)$ in a pure imaginary case for the elastic scattering amplitude, which we consider here for simplicity has been written in the following form

$$
U(s, b)=i \tilde{U}(s, b)=i g(s) \exp (-M b / \zeta),
$$


where the factor $g(s)$ increases at large values of $s$ like a power of energy:

$$
g(s)=\left[1+\alpha \frac{\sqrt{s}}{m_{Q}}\right]^{N}
$$

$M$ is the total mass of $N$ constituent quarks with mass $m_{Q}$ in the initial hadrons and parameter $\zeta$ determines a universal scale for the quark interaction radius without flipping its spin, i.e. $r_{Q}=\zeta / m_{Q}$.

To evaluate asymmetry dependence on $x_{F}$ and $p_{T}$ the semiclassical correspondence between transverse momentum and impact parameter has been used. Integrating by parts, we can rewrite the expression for the asymmetry in the form:

$$
A_{N}^{\pi^{0}}(s, \xi) \simeq x_{F} r_{Q}^{f l i p} \frac{M}{3 \zeta} \frac{\int_{0}^{\infty} b d b I_{0}(s, b, \xi) \tilde{U}(s, b) /[1+\tilde{U}(s, b)]^{3}}{\int_{0}^{\infty} b d b I_{0}(s, b, \xi) /[1+\tilde{U}(s, b)]^{2}},
$$

At small values of $b$ the values of $U$-matrix are large, and we can neglect unity in the denominators of the integrands. Thus the ratio of the two integrals (after integration by parts of nominator in Eq. (11)) is of order of unity, i.e. the energy and $p_{T}$-independent behavior of asymmetry $A_{N}^{\pi^{0}}$ takes place at the values of transverse momentum $p_{T} \gg x_{F} / R(s)$ :

$$
A_{N}^{\pi^{0}}(s, \xi) \simeq x_{F} r_{Q}^{f l i p} \frac{M}{3 \zeta}
$$

This flat transverse momentum dependence of asymmetry results from the similarity of the rescattering effects for the different spin states, i.e. spin-flip and spin-nonflip interactions undergo similar absorption at short distances and the relative magnitude of this absorption does not depend on energy. It can be considered as a one of the manifestations of the unitarity. The numerical value of polarization $A_{N}^{\pi^{0}}$ can be significant. Indeed, there is no small factor in (12). The function $R(s)$ is the hadron interaction radius ( $R(s) \sim \ln s$ ), the typical numerical falue of $R$ has been taken to be equal to $1 \mathrm{fm}$. Thus, the typical value of $x_{F} / R(s)$ is $0.1 \mathrm{GeV} / \mathrm{c}$ and the Eq. (12) which is valid at $p_{T} \gg x_{F} / R(s)$, should be applicable in the region $p_{T}>1 \mathrm{GeV} / \mathrm{c}$. The value of $r_{Q}^{\text {flip }}$ is of order $\sim 10^{-1} \mathrm{fm}$ on the basis of the model estimates [6, 8, 11]. The radius of quark interaction $r_{Q}^{\text {flip }}$ responsible for the transition $Q_{\uparrow} \rightarrow Q_{\downarrow}$ changing quark spin. The linear increase of asymmetry with $x_{F}$ follows from the above considerations which, of course, are approximate and valid at $x_{F}$ around unity. Therefore, at smaller values of $x_{F}$ the linear dependence is distorted.

Thus, Eq. 12 is valid in the region of large $x_{F}$. The flat dependence of asymmetry on $p_{T}$ provided by this relation is consistent with the new data from RHIC (we have used data available at the largest values of $x_{F}$ due to approximation made in the model and discussed above) [4, 5] (cf. Fig. 2,3). Comparison 


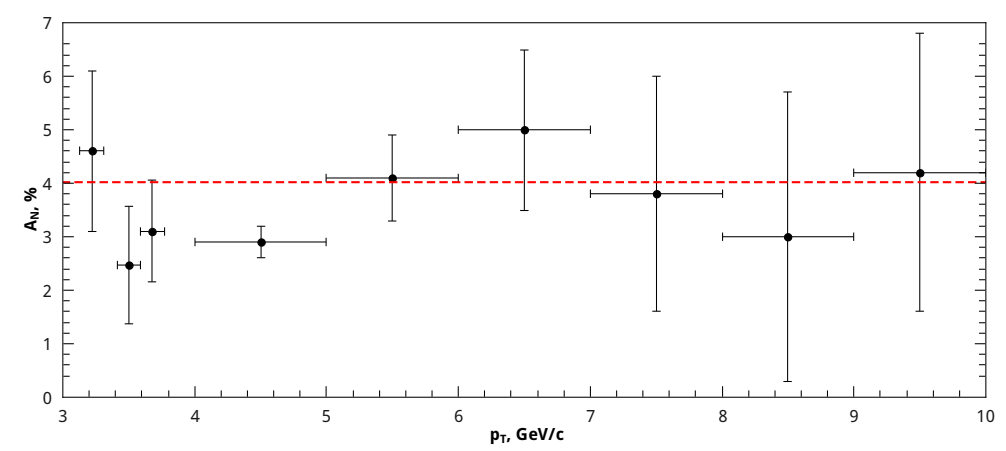

Figure 3: $p_{T}$-dependence of the asymmetry $A_{N}$ in the process $p_{\uparrow}+p \rightarrow \pi^{0}+X$ at RHIC, preliminary data from [4, 5] correspond to pion isolation of $70 \mathrm{mR}$, $\sqrt{s}=500 \mathrm{GeV}$ and $0.32<x_{F}<0.40$.

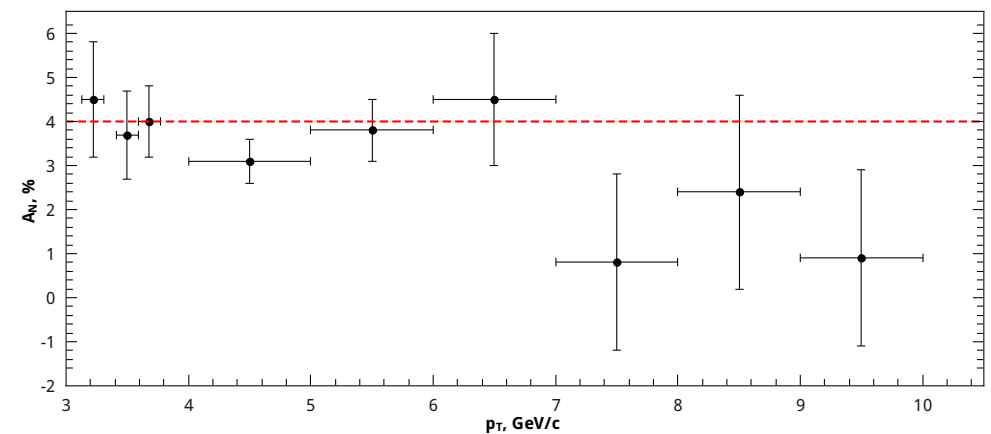

Figure 4: The same plot as in Fig.3, but with data corresponding to pion isolation of $30 \mathrm{mR}$.

with the data allows one to estimate the value of $r_{Q}^{\text {flip }}$ more precisely, namely $r_{Q}^{f l i p} \simeq 0.05 \mathrm{fm}$. Similar mechanism generates SSA in the inclusive production of charged pions. It should be noted that dependencies of SSA on $p_{T}$ consistent with the flat ones have also been observed at lower values of $x_{F}$, namely, in the two regions $0.16<x_{F}<0.24$ and $0.24<x_{F}<0.32$ [4, 5]. The data demonstrate increase of SSA with $x_{F}$. Due to limitation of the model for the large $x_{F}$ region, we have not used those data, but, in principle, the model is in agreement with them too. To be honest, one should not compare the predictions of the model which are valid for $x_{F}$ in the vicinity of unity with the experimental data wich have highest value of $x_{F}$ around 0.4. However, the data demonstrate similar flat dependence on transverse momentum in the the rather wide range $x_{F}$ from 0.16 to 0.4 . We therefore assume that the similar dependence will be valid at $x_{F}$ values around 
unity and perform comparison with the experiment. It should be clarified, that the above agreement with the data has a qualitative value only and for the quantitative analysis the data at higher values of $x_{F}$ are required.

The mechanism of chiral quark fluctuation in the effective field with spin flip is suppressed compared to the direct elastic scattering of quarks and, therefore, it should not play a significant role e.g. in the reaction $p p_{\uparrow} \rightarrow p X$ in the fragmentation region, but evidently it is not the case for the reaction $p p_{\uparrow} \rightarrow n X$. The above features can be observed experimentally: asymmetry $A_{N}$ is consistent with zero for proton production and significantly deviates from zero for the neutron production in the forward region.

In ref. [14] an important issue has been raised, namely a model when trying to explain spin asymmetries should simultaneously describe the data for the unpolarized inclusive cross-sections. In this approach with the effective degrees of freedom - constituent quarks and Goldstone bosons - unpolarized inclusive cross-section at high transverse momenta fllows a generic power-like dependencies on $p_{T}$. At high $p_{T}$ the power-like dependence $p_{T}^{-n}$ with $n=6$ takes place. It originates from the singularity at zero impact parameter $b=0$. The exponent $n$ does not depend on $x_{F}$. This $p_{T}^{-6}$-dependence of the unpolarized inclusive cross-section is consistent with the respective data dependence on the transverse momentum (Fig. 5) [6].

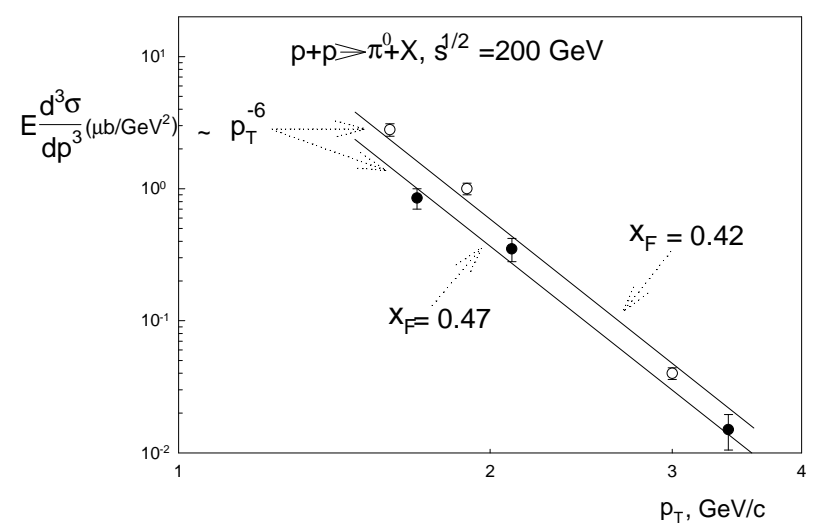

Figure 5: Transverse momentum dependence of unpolarized inclusive crosssection, experimental data from [9].

\section{Conclusion}

It was shown that prediction of the spin filtering mechanism [6] on the flat $p_{T^{-}}$ dependence of single-spin asymmetries is consistent with the new experimental 
data from RHIC. As it often happens, this interpretation is not unique. Such flat dependence can result from finite size of a constituent quark and a presence of the orbital angular momentum of the current quarks residing inside the constituent one [12, 13]. This mechanism is based on the similar ideas as the spin filtering one but is, in principle, different and has no predictive power for $x_{F}$ dependence of SSA. Thus, we would like to treat the seemingly flat $p_{T}$-dependence of SSA in favor of the spin filtering mechanism, while the presence of the internal orbital momentum in the structure of constituent quarks still remains to be an interesting option and cannot be excluded at the moment (cf. e.g. [15]).

Finally, we would like to stress again that the experimental data set [4, 5] is the preliminary one and we hope that the final data will show smaller error bars and allow one to provide a quantitative discrimination of the model predictions for SSA.

\section{Acknowledgment}

We are grateful to Yuri Kovchegov for the information on the new experimental data from RHIC and the interesting discussions.

\section{References}

[1] Y.V. Kovchegov, M.D. Sievert, Phys. Rev. D 86 (2012) 034028.

[2] A. Metz, D. Pitonyak, arXiv:1212.5037v1, 2012.

[3] L. Pondrom, Phys. Rep. 122 (1985) 57.

[4] S. Heppelmann (for STAR Collaboration), Talk at 2012 RHIC and AGS Annual Users' Meeting, BNL, Upton, June 12-15, 2012.

[5] G. Igo, Talk at the Interanational Workshop on Diffraction in High Energy Physics (Diffraction 2012), Puerto del Carmen, Lanzarote, Canary Islands, Spain, September 10-15, 2012.

[6] S.M. Troshin, N.E. Tyurin, Phys. Part. Nucl. 41 (2010) 54.

[7] H. Georgi, A. Manohar, Nucl. Phys. B 234 (1984) 189.

[8] S.M. Troshin, N.E. Tyurin, Phys. Rev. D 49 (1994) 4427.

[9] J. Adams et al.(STAR Collaboration), Phys. Rev. Lett. 92 (2004) 171801.

[10] T.P. Cheng, L.F. Li, Phys. Rev. Lett. 80 (1998) 2789.

[11] S.M. Troshin, N.E. Tyurin, Z. Phys. C 45 (1989) 171. 
[12] S.M. Troshin, N.E. Tyurin, Phys. Rev. D 52 (1995) 3862.

[13] V.V. Mochalov, S.M. Troshin, A.N. Vasiliev, Phys. Rev. D69 (2004) 077503.

[14] C. Bourrely, J. Soffer, Eur. Phys. J. C 36 (2004) 371.

[15] F. Arash, Talk at the Interanational Workshop on Diffraction in High Energy Physics (Diffraction 2012), Puerto del Carmen, Lanzarote, Canary Islands, Spain, September 10-15, 2012. 